\title{
Article
}

\section{Hindered settling of particulate dispersions}

Mercer, Tim, Bissell, Philip, Gotaas, James Alan and Gilson, Ray G. Available at http://clok.uclan.ac.uk/31996/

Mercer, Tim ORCID: 0000-0002-1557-2138, Bissell, Philip ORCID: 0000-00028024-1757, Gotaas, James Alan and Gilson, Ray G. (1999) Hindered settling of particulate dispersions. Journal of Magnetism and Magnetic Materials, 193 (1-

3). pp. 284-287. ISSN 0304-8853

It is advisable to refer to the publisher's version if you intend to cite from the work. http://dx.doi.org/10.1016/s0304-8853(98)00460-0

For more information about UCLan's research in this area go to

http://www.uclan.ac.uk/researchgroups/ and search for < name of research Group>.

For information about Research generally at UCLan please go to http://www.uclan.ac.uk/research/

All outputs in CLoK are protected by Intellectual Property Rights law, including Copyright law. Copyright, IPR and Moral Rights for the works on this site are retained by the individual authors and/or other copyright owners. Terms and conditions for use of this material are defined in the policies page.

\section{CLoK}

Central Lancashire online Knowledge www.clok.uclan.ac.uk

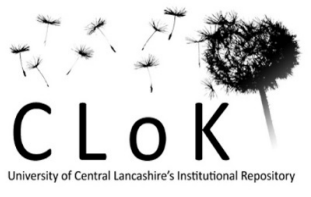




\title{
Author Accepted Manuscript
}

\section{Hindered settling of particulate dispersions}

\author{
T. Mercer ${ }^{\text {a }}$, P.R. Bissell ${ }^{\text {a }}$, J.A. Gotaas ${ }^{\text {a }}$ and R.G. Gilson ${ }^{\text {a }}$ \\ ${ }^{a}$ Department of Physics, Astronomy \& Mathematics, University of Central Lancashire, Preston \\ PR1 2HE, U.K.
}

\begin{abstract}
A computer simulation has been developed that models hindered settling in particulate recording media dispersions. The resulting effective pigment concentration profiles compare well with experimental observations carried out using a scanning column magnetometry technique. Rigorous testing for mass conservation gives added confidence in the feasibility of this model as an aid in the study of suspension structure. As this structure is important in the media manufacturing processes of coating, orientation and drying, these investigations offer the potential to enhance the qualities of the final product.
\end{abstract}

Keywords: Hindered settling; Kozeny-Carman equation; Particulate dispersions; Magnetic recording media

Corresponding author: T. Mercer, Magnetic Materials Research Group, Department of Physics, Astronomy \& Mathematics, University of Central Lancashire, Preston PR1 2HE. , U.K.

Tel: +44-1772-893579, Fax: +44-1772-892903, email: t.mercer1@uclan.ac.uk. 


\section{Introduction}

The properties of dispersions used to produce particulate recording media determine the 'quality' of the final product via a series of further wet state manufacturing processes [1]. Structures formed by the magnetic pigment suspended in a resin-solvent base are important because they are subjected to the mechanics associated with these processes of coating, orientation and drying. The structures are a function of the milling technique necessary for uniform dispersion production as well as the actual material properties and chemical formulation. The ability to characterise these structures is therefore highly relevant to the media manufacturing industry. Combined with dispersion rheology and solvent evaporation data, knowledge of structural information enhances the understanding of the science behind the production process and hence offers the potential for new and improved products.

The opaque, paint-like nature of the dispersion renders 'in-situ' microscopy studies impractical. Real systems also require additional components such as anti-static agents, abrasive agents, lubricants and cross linking agents [2], making microscopy even more difficult. A nondestructive scanning column magnetometry method developed within our group [3] is being used to investigate suspension structure. By observing gravity sedimentation of the magnetic pigment over time there is the potential to measure the structure's specific surface area using hindered settling theory. In this paper we report on the development of a computer simulated model based on this theory that will complement the experimental work.

\section{Experimental details}

Dispersions of a commercial $300 \mathrm{Oe} \gamma-\mathrm{Fe}_{2} \mathrm{O}_{3}$ formulation were produced 'in-house' using standard premixing and milling techniques. Premixing was carried out in a DISPERMAT ${ }^{\circledR}$ water cooled chamber using a saw-tooth blade. Milling and letdown were carried out in a DISPERMAT ${ }^{\circledR}$ SL-C bead mill using $\sim 1 \mathrm{~mm}$ diameter zirconia beads. The standard formulation with a magnetic pigment volume concentration (PVC) of $5.41 \%$ was varied to produce samples within the range $3 \%$ to $9 \%$ by adding different amounts of letdown solution to the stock milled slurry formulation. 
Profiles of pigment concentration against settling column height were obtained using a scanning column magnetometer. Here a column of magnetic dispersion is driven down through the otherwise empty core of a detection coil by a computer controlled stepper motor. The coil forms the inductive part of a tuned Colpitts circuit. Introducing magnetic material into the coil core changes the detection system inductance and causes a corresponding change in the circuit resonant frequency. The field generated by the coil is such that measurements are made in the linear region of the suspensions magnetisation curve. This ensures that the shift in induction is directly proportional to the change in the coil core susceptibility. Also, in particulate systems the magnetic susceptibility of the pigment is linearly dependant on its concentration [4]. However, any ongoing particle agglomeration would be expected to effectively change the system's particle size and this relationship would then no longer hold. Therefore, providing there is no post milling agglomeration, a plot of frequency shift (with respect to the sample free value) as a function of position represents a dispersion's pigment concentration profile.

For the settling observations, samples of dispersion were kept vertical in glass tubes of $\sim 1.1$ $\mathrm{cm}$ internal diameter. The tubes were sealed and held at a constant temperature of $(20 \pm 1){ }^{\circ} \mathrm{C}$ between scans by immersion of the sample chamber in a water bath.

\section{Laminae concept computer model}

The basic concept of model is to divide the column of dispersion up into a series of imaginary laminae. Within each layer the net solids flux, $J=\left(J_{\text {in }}-J_{\text {out }}\right)$ and the resultant change in mass are calculated for time interval $\Delta t$. This is repeated over the suspension height from layer $i=1$ to $n$ to represent the complete column settling within that $\Delta t$. The lamina concept and settling algorithm are illustrated in Fig. 1. Consideration of pigment concentration $C$ (mass per unit volume) along with its settling velocity $Q$ allows a general solids flux flowing across a horizontal plane of area $A$ to be expressed as $j=C Q$. The change in mass of $j A \Delta t$ results in a new pigment concentration of

$$
C(t)=C(t-1)+\frac{j(t-1) \Delta t}{\ell}
$$


within each layer cell of depth $\ell$. In specific terms a net flux $J$ must be calculated as shown in Fig. 1 and be substituted for $j$ in equation (1). The minimum flux condition shown in the algorithm ensures that the rate of mass transport is effectively limited to that of the layer below. This allows material to build up within a cell whilst conserving mass throughout the system.

For concentrated systems (nominal PVC $\geq 10 \%$ ) the fall of any one particle is hindered by the presence of surrounding particles. This allows the suspension to be thought of as a granular packed bed fluidised by an upward flow of liquid. A permeability theory that successfully describes this situation is that of Kozeny-Carman [5-8] with a useful form [9] given as

$$
Q=\frac{1}{k} \frac{\left(\rho_{p}-\rho_{f}\right) g}{\eta S_{v}{ }^{2}}\left(\frac{\varepsilon^{3}}{1-\varepsilon}\right)
$$

Here $k$ is a system dependant constant, $g$ the acceleration due to gravity, $\eta$ the free fluid viscosity, $\rho$ the particle and fluid densities, $\varepsilon$ the void fraction (1-PVC) and $S_{V}$ the specific volume surface area. Hence, the settling rate determined from (2) is used to find $j$ in the algorithm.

\section{Results and discussion}

A series of test runs to check the validity of the model were carried out, producing the typical concentration profiles of Fig. 2. The plot compares profiles of the same sample over many iterations (time) and so allows settling effects to be observed. The maintenance of a shear step at the top of the column is expected in hindered settling experiments due to the fact that the particles fall 'en-bloc' at an initially constant rate $Q$. This leads to three distinct regions being formed in the tube. A near solids free fluid supernatant to a contracting plug occupies the top layers. The plug descends in the middle region of the column until it is eventually subsumed by the build up of material in the bottom compaction layers. The decrease in $Q$ at this point can be clearly seen here on profiles of equal time spacing. A plot of the total pigment mass as a function of the number of iterations can be seen in the inset of Fig. 2. with each data point obtained from the area under the corresponding curve of the main figure. Mass conservation has clearly been achieved and does not vary by more than $\pm 0.6 \%$ from the normalised value. 
To aid comparison with experiment the model was extended by numerically sweeping the known scanning column magnetometer coil response or 'form factor' over the dispersion height. Also, the initial work tried to account for the maximum PVC attainable within a given system by imposing a concentration limit of $(1-\varepsilon)<1$ that resulted in the step observed at $A$ in the bottom layers of Fig. 2. This was found to be unrealistic in terms of Kozeny-Carman (2) due to the effective solids ratio requirement of $(1-\varepsilon)=1$ for zero flow. The resultant settling profiles over time are shown in Fig. 3(a) with the inset plot of the SCM coil response included for completeness. The curves compare well with the experimental data of Fig. 3(b), reproducing all the essential features observed empirically. This was achieved by adjustment of the model PVC until the profile morphologies matched well and in all cases this resulted in a value for the PVC of about $60 \%$. Initially this seems unrealistic in comparison to the PVC of order $5 \%$ for the standard dispersion formulation. However, magnetostatic attraction is expected to cause some clustering of the particles within a real system and as chemically induced association (flocculation) has been seen to increase the effective particle volume by a factor of approximately 8 [10], this does not seem unreasonable. Indeed, the particle's 'coat' of surfactant in magnetic media dispersions would exaggerate this effect further.

The equivalent free fluid channel width implied by the porosity $(\varepsilon)$ results obtained here may now be used in drying studies. Work is planned to extend an existing drying model to include these findings. Rheological investigations are currently in progress so that hindered settling theory may be used to find the specific volume surface area of the suspension and the equivalent Stokes' particle size. The effects of these parameters on coating and orientation can then be studied.

\section{Conclusions}

A computer simulated laminae model of the hindered settling of magnetic media dispersions has been successfully produced to complement experimental studies. The algorithm conserves mass and the resulting concentration profiles compare well with those of experimental systems produced by a scanning column magnetometry technique. Further studies should improve the understanding of structures within the wet stages of the media production process and offer the potential to enhance the properties of the final product. 


\section{References}

[1] G.N. Coverdale, R.W. Chantrell, A. Satoh, and R. Vietch, J. Appl. Phys. 81 (1997) 3818

[2] K. O'Grady, R.G. Gilson, and P.C. Hobby, J. Magn. Magn. Mater. 95 (1991) 341

[3] P.M. Sollis, P.R. Bissell, T. Mercer, J.A. Gotaas, and R.G. Gilson, J. Magn. Magn. Mater. 177 (1998) 892

[4] R.W. Chantrell, J. Popplewell, and S.W. Charles, IEEE Trans. Magn. 14 (1978) 975

[5] J. Kozeny, Sitzber. Akad. Wiess. Wien, Math. -naturw. Kl. Abt. IIa 136 (1927) 271

[6] P.C. Carman, Trans. Inst Chem. Engrs. 15 (1937) 150

[7] P.C. Carman, J. Soc. Chem. Ind. 57 (1938) 225

[8] P.C. Carman, J. Soc. Chem. Ind. 58 (1939) 1

[9] J. Azizi and D. Dollimore, Ohio Journal Of Science 86 (1986) 22

[10] J.I. Bhatty, L. Davies, D. Dollimore, and A.H. Zahedi, Surface Technology 15 (1982) 323 


\section{Figure captions}

Fig. 1. Schematic of the settling algorithm showing the calculations carried out and the top to bottom order. Each layer's mass flux is limited to that of the layer below which ensures that mass is conserved throughout the system.

Fig. 2. Test run on laminae model concentration profile. No account of convolution with the experimental equipment is calculated here. The inset shows conservation of mass over many iterations with the data points obtained from the area under each curve of the main plot.

Fig. 3. Concentration profiles of (a) the laminae model convolved with the SCM coil response of the inset and (b) an experimental sample of $\gamma-\mathrm{Fe}_{2} \mathrm{O}_{3}$ measured using a scanning column magnetometer. An effective solids concentration of $60 \%$ was required in the model to obtain the reasonable comparison shown. 
Fig. 1., T. Mercer, TP2-2

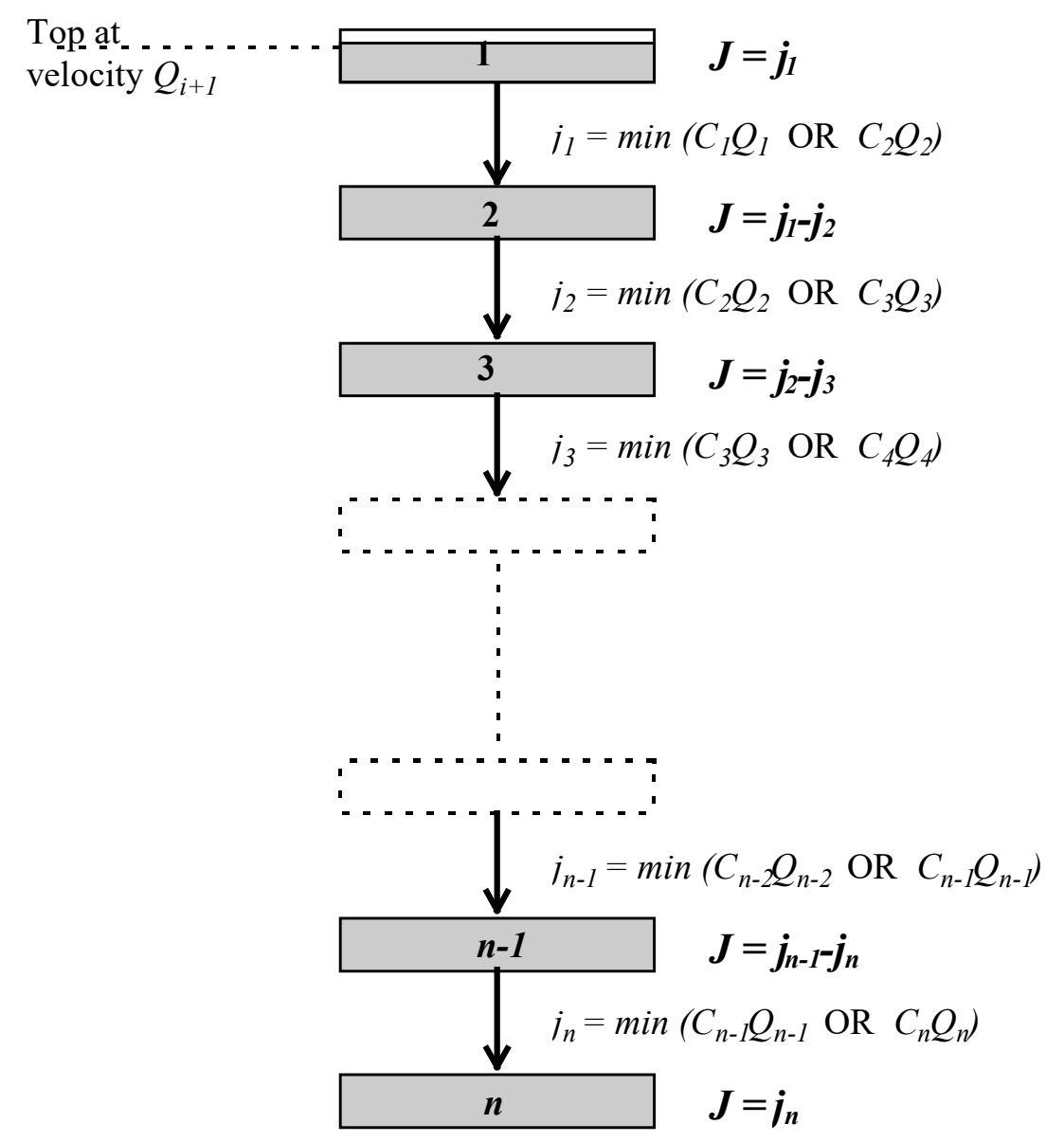


Fig. 2, T. Mercer, TP2-2

Fig. 2

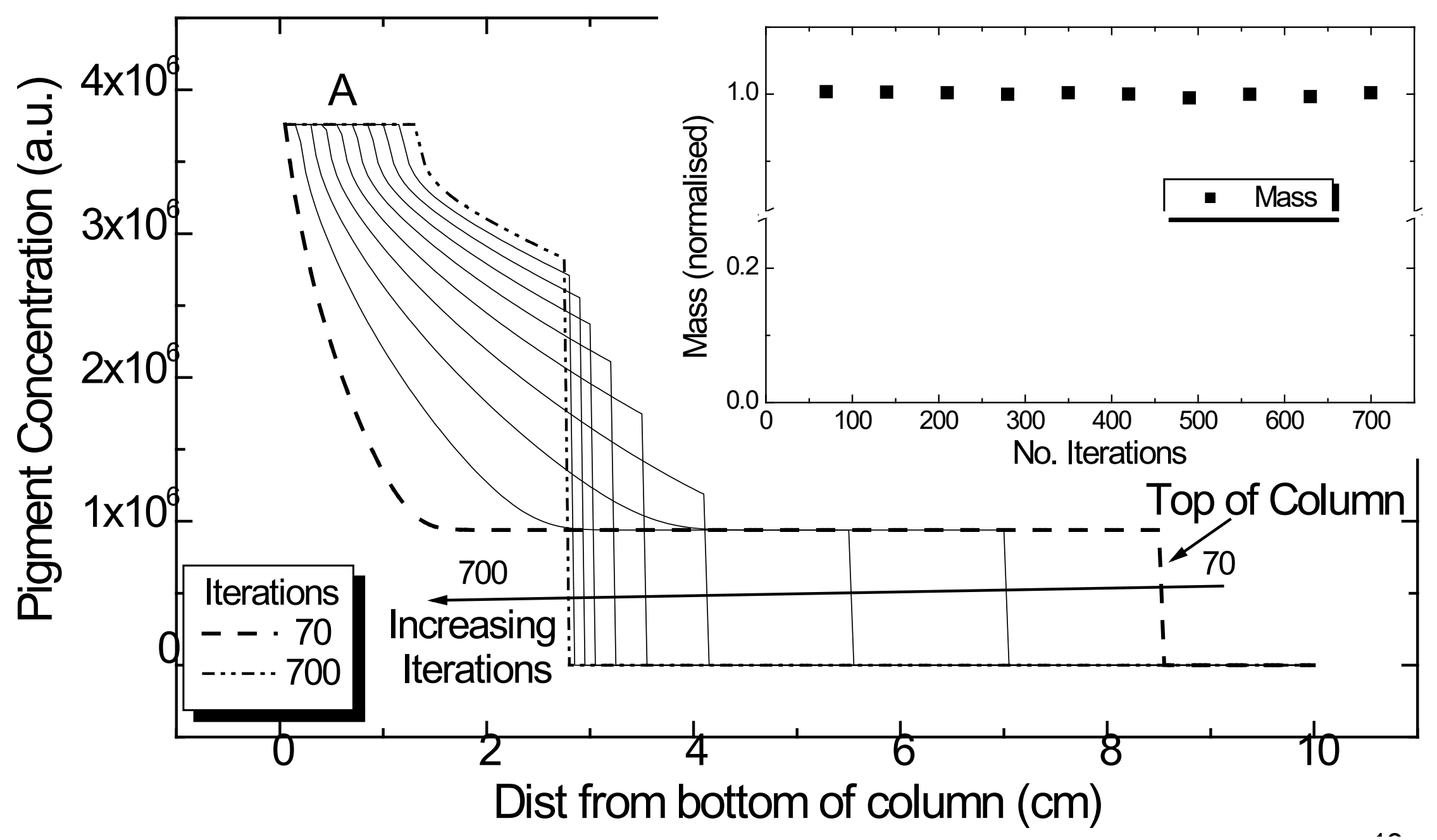


Fig. 3., T. Mercer, TP2-2

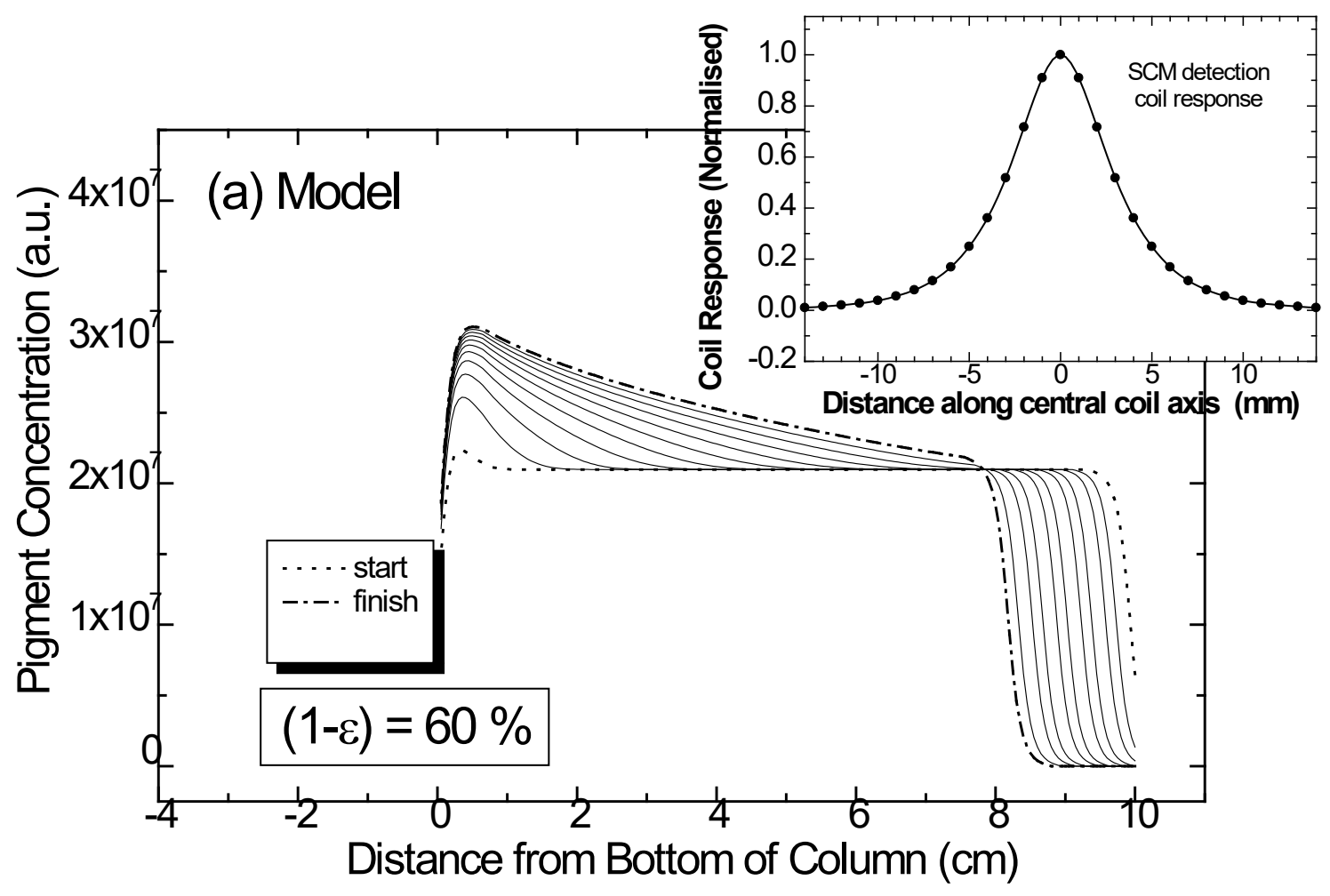

Fig. 3(b), T. Mercer, TP2-2

Fig. $3(b)$

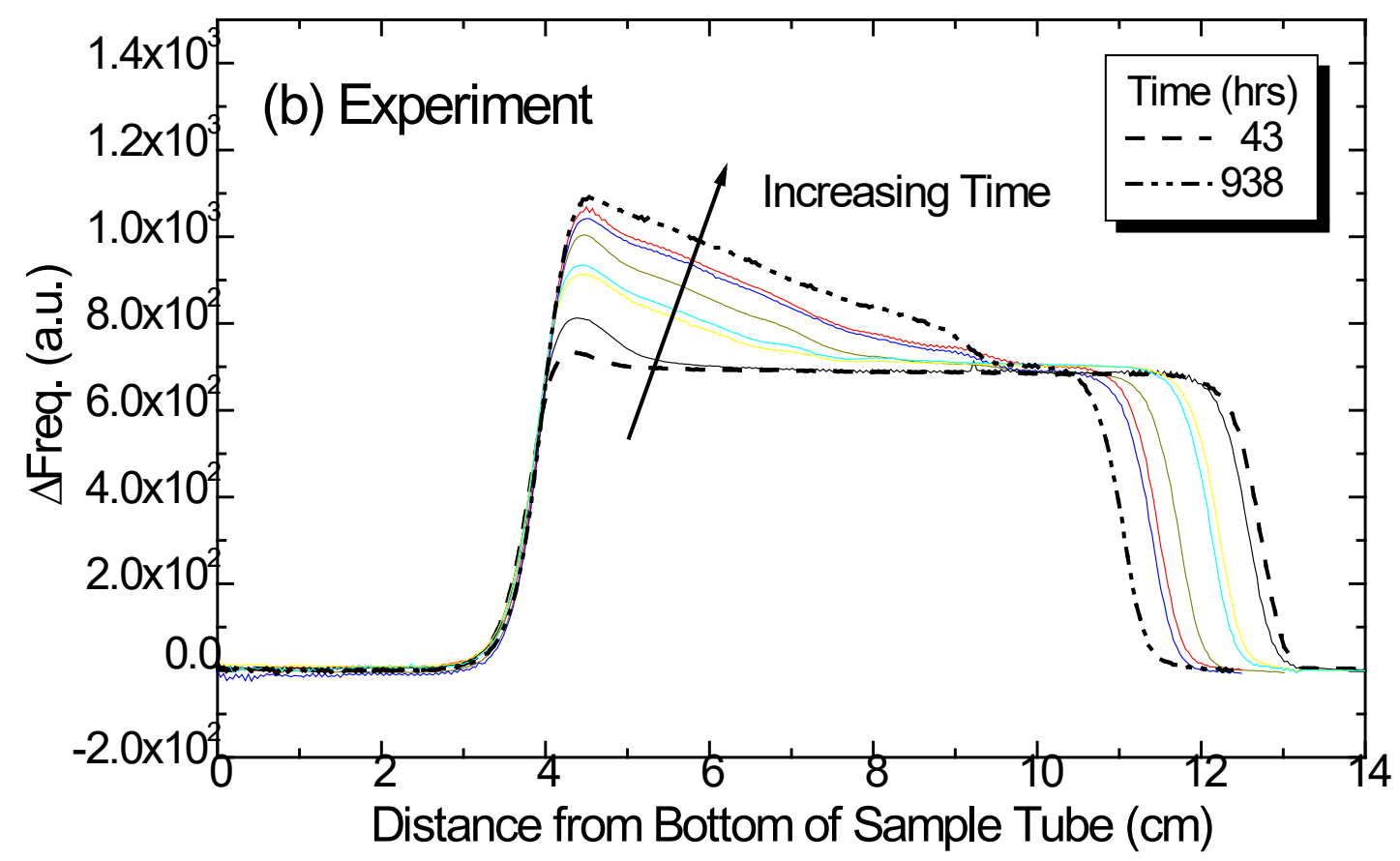

